\title{
THE EFFECTS OF ROUTE GUIDANCE ON SPATIAL LEARNING
}

\author{
Yi-Fang Tsai \& Matthew Peterson \\ George Mason University \\ Fairfax, Virginia, USA \\ Email: ytsai1@masonlive.gmu.edu
}

\begin{abstract}
Summary: Participants engaged in five driving routes while performing a secondary PDT task. For each route, participants drove to four distinct destinations. Route guidance was given to all subjects to help guide them to their destinations. Most of the participants were able to complete each driving route within the allotted time of 10 minutes. The drivers had repeated exposures to the locations and routes between the destinations and were told they would be asked the whereabouts of the destinations at the end of the driving routes. Overall, the participants had difficulty explicitly marking the locations of the destinations on a map, and indicating directional relationship between buildings. PDT performance and lane deviation stayed consistent across driving route tasks. The participants performed best at naming the street locations where the buildings were located. The use of route guidance on the ability to process navigation information may suppress cognitive map formation.
\end{abstract}

\section{INTRODUCTION}

Route guidance is known to aid drivers in making more efficient travel choices and in navigating unfamiliar environments. Route guidance is generally referred to as prescriptive information designed to direct a driver along a prescribed path to their destination (Adler, 2001). Technologies with route guidance are also increasingly pervasive as more nomadic and invehicle GPS systems are readily available and affordable.

Spatial learning is described as progressing through stages of development, mainly by landmark, route, and survey knowledge. Landmark knowledge is a memory for distinctive objects such as a particular building along a road. Route knowledge is the procedural linking of these objects, as well as forming distance and direction estimates. Survey knowledge (Siegel \& White, 1975) is the ultimate integration of these landmarks and routes into a cognitive map of the environment. Route guidance systems have been hypothesized to speed up the process of route knowledge (Adler, 2001; Schofer, 1993).

The effects of repeated route guidance use on driver behavior are not well understood. The goal of this study is to test the effects of route guidance on driver performance and route-learning. Spatial learning can occur from direct experience, using maps, or receiving and following verbal or written instructions. As a driver's spatial knowledge of the environment increases, the ability to make route choices and perform high level tasks like linking together landmarked locations can occur. The objective of this study is also to test whether driving and secondary task performance improves over repeated route sessions. 
In this study, participants performed simulated driving routes to four destinations. All drivers are given route guidance to assist them to reach their destinations. While the locations of the starting point and the destinations are always the same, the order of the four destinations are randomized. As a secondary task, participants responded to a peripheral detection task. This measure is commonly used to assess workload (Jahn et al., 2005). Improvement in driving performance is predicted over the repeated sessions. Route guidance is predicted to help drivers better navigate their driving environment and learn the locations and routes to the four destinations.

\section{METHODS}

\section{Participants}

The 20 participants ranged in age from 18 to 30 years old $(\mathrm{M}=21.85, \mathrm{SD}=0.72,10 \mathrm{men} ; 10$ women). All participants were at least 18 years of age and held a valid driver's license. Participants were either given a Sona subject pool credit hour or \$15 for their participation.

\section{Equipment}

A Dell Dimension 2.13 GHz Intel Core 2 DM061 computer running SimCreator software (Realtime Technologies, Inc.) was used to present the driving simulation. The roadway environment and driving tasks were developed in RTI's SimVista authoring tool. The driving task stimuli was presented at $60 \mathrm{~Hz}$ on a 20.1" widescreen LCD monitor. The screen resolution was 1680 x 1050. A Logitech Driving Force GT steering wheel and pedals with force feedback was also used for controlling the simulated vehicle. The peripheral detection task stimuli was presented at $60 \mathrm{~Hz}$ on a 17” CRT Monitor at a screen resolution of 1024 x 768.

\section{Navigation Driving Task}

Each participant completed five driving route tasks between four destinations: Walgreens, KFC, Blockbuster, and SouthTrust Bank. The order of the destinations were randomized for each route. Participants were given up to 10 minutes to complete each driving route task. A compass showing the directional heading $(\mathrm{N}, \mathrm{S}, \mathrm{E}, \mathrm{W})$ of their vehicle was located on the upper left-hand side of the screen. All routes included voice guidance preceding each turn. In addition to the auditory cue, the directions also appeared at the center of the screen (e.g. "Turn right on A St."). Figure 1 shows a map of the world where the locations of the buildings are marked. The driving world was created using SimVista. The layout of the driving world was designed in a grid-like pattern, where numbered streets were arranged from North to South and lettered streets were arranged from East to West. The street name was posted on green signs along the roadway such that the driver could always see the street name that they were currently on. There was sparse ambient traffic on the roadway. A light density of residential and commercial buildings was also present along the roadside. 


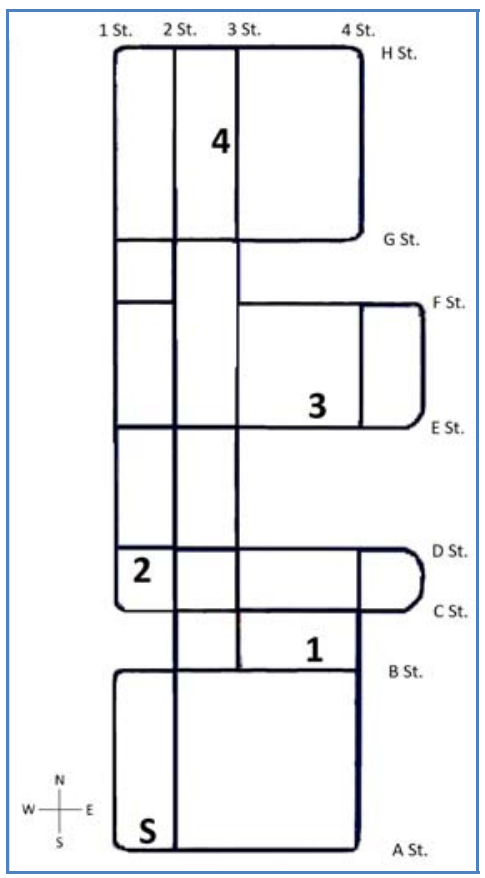

Figure 1. Layout of Driving World

(S=Start, 1=Walgreens, 2=SouthTrust Bank, 3=Blockbuster, 4=KFC)

\section{Peripheral Detection Task}

The secondary task in this study was a modified peripheral detection task (PDT). This task was used in this study as a loading task and also a workload measure. Studies have shown that peripheral detection is sensitive to route demands (Jahn et al., 2005). The standard task required a manual response to stimuli presented at eccentricities between $5^{\circ}$ and $25^{\circ}$ left of the drivers' normal line of sight (van Winsum, Martens, \& Herland, 1999). The stimuli in our task consisted of a red dot or blue dot visible for 1-2 seconds presented at an interval of 3-5 seconds. A red dot appeared in $80 \%$ of the trials. The dot either appeared at a near location of approximately $15^{\circ}$ left of the line of sight of the driver or at a far location approximately $21^{\circ}$ left of the line of sight of the driver. The participant responded by pressing a button only when a red dot appeared on the screen.

\section{Procedure}

Participants were instructed to learn and complete five driving routes to the best of their ability between four destinations: Walgreens, KFC, Blockbuster, and SouthTrust Bank. Participants were told that all routes have voice guidance where a visual and auditory cue (e.g. "Turn right on A St.”) is given prior to a turn. They were also told that the voice guidance system would not recalculate and to make a u-turn if a turn is missed. The drivers were instructed that they would be asked about the locations of the destinations after the five routes are completed. After receiving instructions, participants practiced driving around the world with the secondary PDT task for approximately 8 minutes. The participants were told that the layout of the world was a grid-like design where numbered streets go from North to South and lettered streets go from East to West. They were instructed that they would always start at the same starting point and the 
locations of the buildings never change. The order of the four destinations was randomized for each route. Participants were also instructed to perform the driving routes as safely as possible as they would normally drive and to perform the secondary PDT as quickly and accurately as possible.

Destination quiz. After completing the driving route tasks, participants were given a paper quiz. In the first part of the quiz, participants were given a map similar to Figure 1 (without the locations 1-4 marked). The participants were told to mark the corresponding numbers for the four destinations on the map, similar to Thorndike \& Golding's (1981) location task.

Performance on this task was measured by the difference between the participants' mark and the actual location of the destination on the map. The second part of the quiz asked participants the relative cardinal directions between locations on the map (e.g. Blockbuster is North/South/East/West of Walgreens). The third part of the quiz asked the street names for the four destinations (e.g. KFC is on St.).

\section{RESULTS}

A repeated measures ANOVA was used to analyze the subjects' driving and PDT performance across driving routes.

Route task completion. A majority of participants completed their driving route tasks to the four destinations. In the first driving route task, $70 \%$ of participants completed the full route, $20 \%$ of participants completed three out of four destinations, and $10 \%$ of participants completed half the route. In the second driving route task, 19 participants (or 95\%) completed the full route and only one participant completed half the route. In the subsequent third and fourth route tasks, $90 \%$ of participants completed the full route and $10 \%$ of participants completed three out of four destinations. In the final driving route task, $85 \%$ of participants completed the full route, $10 \%$ of participants completed three out of four destinations, and one of participants (or 5\%) completed half the route. Route completion was affected by sex, $t(18)=2.26, p=0.04$. Males had a higher average of route completion $(M=99.50 \%, S D=0.02)$ compared to females $(M=91.50 \%, S D=0.11)$.

PDT performance. Participants' performance on the PDT task across the five routes were not significantly different, $F(4,19)=0.23, p=0.92$. The average response time to a red dot in the PDT task was $1229.34 \mathrm{~ms}(S D=78.66)$. PDT performance was not affected by sex, $t(18)=$ $1.22, p=0.24$. Participants rarely responded to the blue dot (no-go stimulus). On average, participants did not respond to a red dot $17 \%$ of the time $(S D=0.03)$ and no significant difference was found across the five routes, $F(4,19)=2.15, p=0.08$.

Driving performance. Average lane position $(M=1.87$ meters, $S D=0.04)$ did not significantly change across route tasks, $F(4,19)=1.85, p=0.13)$. Average speed, however, significantly increased from the first route $(M=25.67 \mathrm{mph}, S D=1.25)$ to the third route $(M=29.80, S D=$ $1.04)$ but decreased and stayed steady for the fourth $(M=28.94, S D=1.19)$ and fifth routes $(M=$ 28.95, $S D=1.06), F(4,14)=10.43, p=0.0001$. Speed stayed consistent across males and females, $t(18)=0.89, p=0.38$. 
Destination quiz performance. The first part of the quiz required participants to locate and mark the four destinations on a map of the driving world. The starting point was given as a reference landmark. The streets on the map were also labelled similar to Figure 1. A score between 0 and $0.5 \mathrm{~cm}$ was considered correctly identifying the location of the destination. Participants, on average, did not perform well on marking the locations on the map but were able to find 1.35 locations $(S D=0.28)$. The closest marked location 1 (i.e. Walgreens) was $1.51 \mathrm{~cm}$ away from the actual location. SouthTrust Bank was marked 2.01cm away from the actual location; Blockbuster was marked $1.80 \mathrm{~cm}$ away from the actual location; KFC was marked the furthest away at an average of $2.78 \mathrm{~cm}$ from the actual location.

Three of the 15 participants found all the locations on the map and had perfect scores on the second directional portion of the quiz, where they were asked about the relative locations of the buildings from each other, and perfect scores on the third portion where they named the streets of where the buildings were located. Participants averaged 58\% on the directional portion of the quiz. Participants performed best on the street naming portion of the quiz where about $45 \%$ of the participants were able to correctly identify all the streets the buildings were located. On average, participants scored $60 \%(\mathrm{SD}=0.09)$ on this task.

\section{DISCUSSION}

One goal of the study was to see how participants' recall of the building locations was affected by route guidance. While participants were able to reach all four destinations, their ability to explicitly recall the destinations were poor. Furthermore, PDT performance and driving performance, specifically lane position, did not change from the first route to the last route. Although overall PDT performance did not improve across subsequent routes, the average participants' vehicle speed did increase over the first routes. This may indicate that the participants were becoming familiar with performing the routes and were more confident in maintaining a faster speed.

One possibility for poor recall may be supported by differences in two possible strategies for learning a route proposed by Aginsky and colleagues (1997). The authors suggest that drivers may follow a visually dominated or spatially dominated strategy. In a visually dominated strategy, participants may visually recognize decision points along a route but they are not integrated into a survey representation. In a spatially dominated strategy, participants represent the environment as a survey map from the start. Scenes and landmarks may be recognized visually but may not be used for navigational purposes. Although all participants received navigational directions during the route, participants may have chosen a particular strategy to learn the locations of the destinations.

A previous driving simulator study by Burnett and Lee (2005) found that participants who navigated around a representation of a small town with a route guidance system were less able to remember routes and developed poorer mental representation compared to those who used a map to plan their route. This effect was attributed to the level of information processing required of the participants where the participants using the map required processing of orientation and directions at a deeper level than the participants who used the route guidance system. This is 
another possibility why participants' performance on the location task (marking location on the map) was worse compared to when asked to name the streets the buildings were located on.

In summary, participants engaged in repeated driving routes to four destinations while performing a secondary PDT task. Route guidance was given to aid all subjects to reach their destinations. Most of the participants were able to complete each driving route within the allotted time of 10 minutes. Although the drivers had repeated exposures to the locations and routes between the destinations, they had difficulty explicitly marking the locations of the destinations on a map, indicating directional relationship between buildings. The participants performed best at naming the street locations where the buildings were located. The use of route guidance on the ability to process navigation information may suppress cognitive map formation. More research is needed to understand the effects long term use of route guidance in vehicle navigation.

\section{ACKNOWLEDGMENTS}

The authors would like to thank the Psychology department of George Mason University for their support with subject payment.

\section{REFERENCES}

Adler, J. L. (2001). Investigating the learning effects of route guidance and traffic advisories on route choice behavior. Transportation Research Part C, 9, 1-14.

Aginsky, V., Harris, C., Rensink, R., \& Beusmans, J. (1997). Two strategies for learning a route in a driving simulator. Journal of Environmental Psychology, 17, 317-331.

Jahn, G., Oehme, A., Krems, J. F., \& Gelau, C. (2005). Peripheral detection as a workload measure in driving: Effects of traffic complexity and route guidance system use in a driving study. Transportation Research Part F, 8, 255-275.

Schofer, J. L., Khattak, A., \& Koppelman, F. S. (1993). Behavioral issues in the design and evaluation of Advanced Traveler Information Systems, Transportation Research Part C, 1(2, 107- 117.

Siegel, A. W. \& White, S. H. (1975). The development of spatial representations of large scale environments. In H. W. Reese (Ed.), Advances in child development and behaviour: Vol. 10 (pp. 955). New York: Academic Press.

Thorndyke, P. W., \& Goldin, S. E. (1981). Ability differences and cognitive mapping skill. (Report No. N-1667-ARMY). Santa Monica, CA: Rand Corporation.

van Winsum, W., Martens, M. H., \& Herland, L. (1999). The effects of speech versus tactile driver support messages on workload, driver behavior and user acceptance. (Report No. TM-99-C043). Soesterberg, Netherlands: TNO Human Factors. 